09

\title{
Получение ультракоротких мощных оптических импульсов от полупроводниковых лазеров за счет управления параметрами токовой накачки
}

\author{
(C) Е.Д. Колыхалова, ${ }^{1,2}$ В.В. Дюделев, ${ }^{1,2}$ C.В. Зазулин, ${ }^{3}$ С.Н. Лосев, ${ }^{1,4}$ А.Г. Дерягин, ${ }^{1}$ В.И. Кучинский, ${ }^{1}$ \\ М.В. Ефанов, ${ }^{3}$ Г.С. Соколовский ${ }^{1,2}$ \\ ${ }^{1}$ Физико-технический институт им. А.Ф. Иофффе РАН, \\ 194021 Санкт-Петербург, Россия \\ ${ }^{2}$ Университет ИТМО, \\ 197101 Санкт-Петербург, Россия \\ 3 ЗАО „ПК „ФИД-ТЕХНИКА“, 194223 Санкт-Петербург, Россия \\ ${ }^{4}$ Санкт-Петербургский политехнический университет Петра Великого, \\ 195251 Санкт-Петербург, Россия \\ e-mail: gs@mail.ioffe.ru
}

(Поступило в Редакцию 24 мая 2017 г.)

Исследованы полупроводниковые лазеры с волоконным выводом излучения при накачке мощными короткими импульсами тока. Показано, что подбор параметров токовой накачки дает возможность получить существенное обострение выходного оптического импульса: менее $80 \mathrm{ps}$ для одномодового лазера и $120 \mathrm{ps}$ для широкополоскового многомодового лазера при длительности импульса накачки $1 \mathrm{~ns}$ и менее $40 \mathrm{ps}$ для одномодового лазера при длительности импульса накачки $0.5 \mathrm{~ns}$.

DOI: $10.21883 /$ JTF.2017.12.45215.2359

На сегодняшний день источники короткоимпульсного оптического излучения с длительностью импульса менее 150 ps и с высокой импульсной мощностью (более $1 \mathrm{~W}$ ) требуются для многих областей науки и техники, таких как лазерная локация, лазерное экспонирование, оптическая связь, нелинейное преобразование частоты, а также генерация сигналов произвольной формы [1-5]. Обычно для получения выходного импульсного излучения с такими параметрами используют режим модуляции добротности в твердотельных и волоконных лазерах. К сожалению данный метод связан с существенными неудобствами в применении, а твердотельные и волоконные лазеры громоздки и дороги. Применение полупроводниковых лазеров является хорошей альтернативой этим подходам. Поэтому получение от таких лазеров мощных импульсов пикосекундной длительности по-прежнему является актуальной задачей. В то же время получение от полупроводниковых лазеров мощных пикосекундных импульсов требует применения импульсного режима накачки, что приводит к влиянию на их Ватт-амперную характеристику сложных динамических эффектов, таких как нетемпературное насыщение усиления [6], задержка включения лазера как с основного, так и с возбужденного квантового состояния $[7,8]$, срыв генерации $[9,10]$, переключение генерации с основного на возбужденное состояние в процессе развития импульса [11] и др.

Настоящая работа посвящена экспериментальному исследованию характеристик выходного оптического излучения, в частности, обострению выходных импульсов промышленных полупроводниковых лазеров при накачке мощными токовыми импульсами наносекундной и субнаносекундной длительности с частотой повторения $1-10 \mathrm{kHz}$ с целью получения от них мощных оптических импульсов пикосекундной длительности. Для формирования импульсов тока использовался генератор на основе мощных быстродействующих полупроводниковых ключей, подробное описание которых приведено в работе [12], а применение для накачки полупроводниковых лазеров представлено в [13]. Были исследованы многомодовые и одномодовые полупроводниковые лазеры. Длина волны генерации многомодовых лазеров находилась в диапазоне 960-980 nm, мощность излучения в непрерывном режиме составляла $10 \mathrm{~W}$ при токе накачки 12 А. Лазеры были оснащены волоконным выводом излучения через многомодовое оптическое волокно с диаметром сердцевины $100 \mu \mathrm{m}$. Длина волны генерации узкополосковых одномодовых лазеров находилась вблизи $1060 \mathrm{~nm}$. Выходная мощность излучения составляла $170 \mathrm{~mW}$ при рабочем токе $300 \mathrm{~mA}$. Вывод излучения осуществлялся через одномодовое оптическое волокно. Следует отметить, что данные образцы полупроводниковых лазеров являлись промышленными корпусированными изделиями и не подвергались дополнительному процессированию для получения коротких импульсов света, предполагающему внесение в резонатор лазерного диода областей с насыщающимся поглотителем для обеспечения режима пичкования лазера [14] или подавления паразитных осцилляций [15]. Регистрация формы оптического импульса производилась при помощи $50 \mathrm{GHz}$ стробоскопического осциллографу Agilent 86100A и быстродействующего фотоприемника Optilab PD-30 с полосой пропускания $30 \mathrm{GHz}$. Следует отметить, 

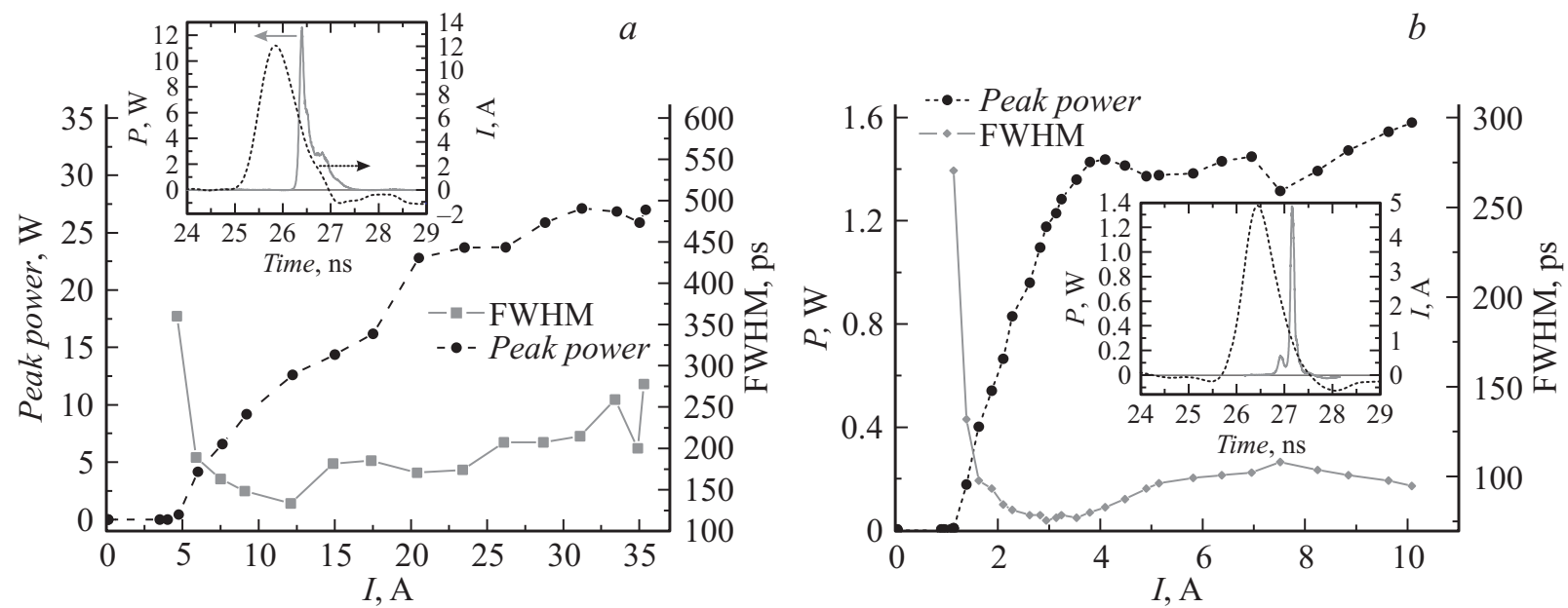

Рис. 1. Зависимости пиковой мощности и полуширины длительности выходного оптического импульса многомодового $(a)$ и одномодового $(b)$ лазера в зависимости от амплитуды импульса тока накачки длительностью менее $1 \mathrm{~ns}$. На вставках представлены осциллограммы импульса тока накачки и выходного оптического импульса при накачке многомодового лазера импульсами тока амплитудой $12.1 \mathrm{~A}(a)$ и $4.1 \mathrm{~A}(b)$.
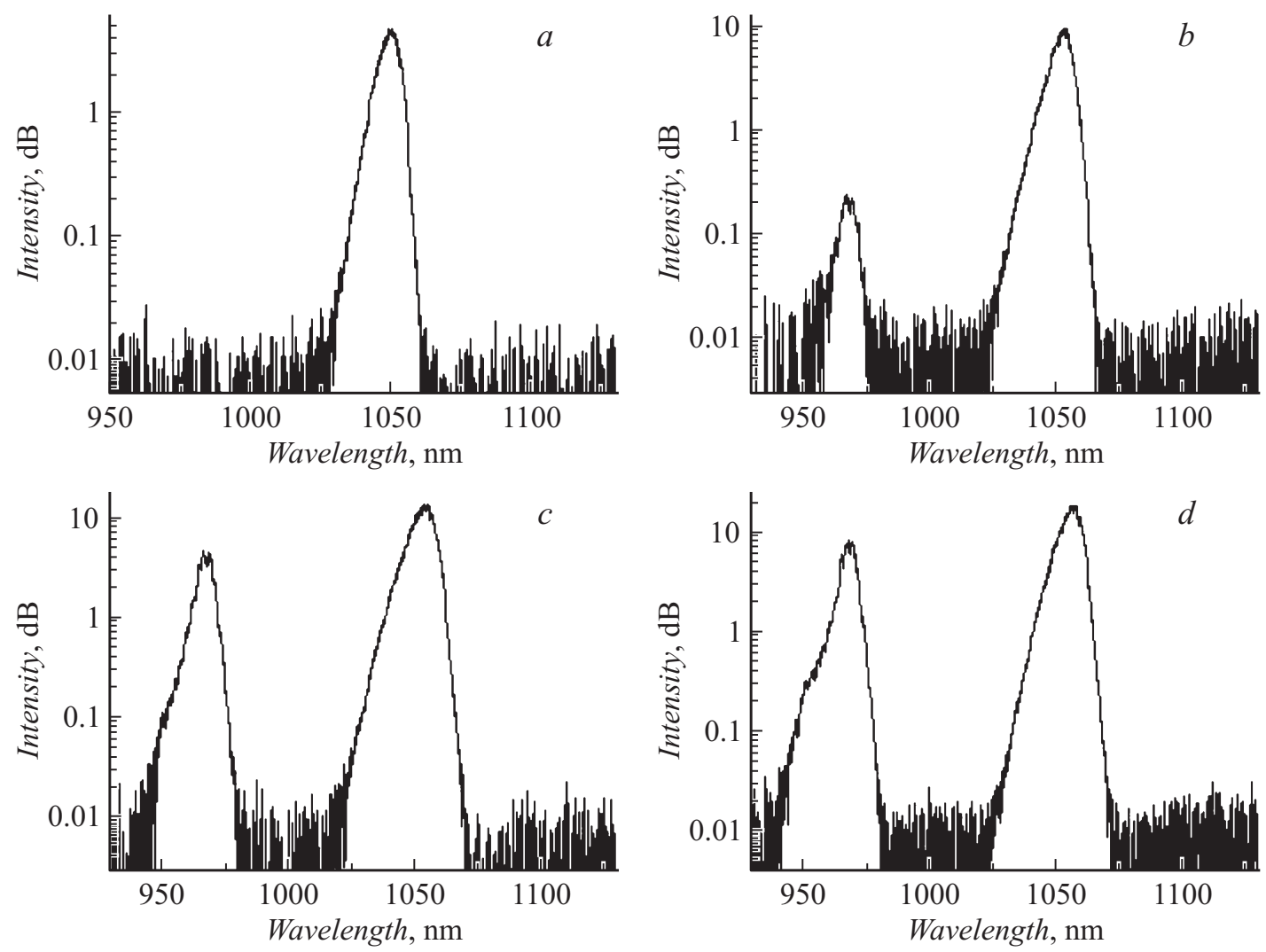

Рис. 2. Спектры выходного излучения полупроводникового одномодового лазера при накачке токовыми импульсами длительностью $1 \mathrm{~ns}$ амплитудой: $a-1.6, b-2.6, c-4.9, d-8.8 \mathrm{~A}$.

что благодаря волоконному выводу излучения исследуемых образцов полупроводниковых лазеров, обеспечивающему эффективное смешивание интенсивности всех мод выходного излучения, мы имели возможность провести корректные измерения, соответствующие среднему распределению интенсивности выходного сигнала.
В ходе экспериментов было обнаружено, что при указанных параметрах токовой накачки все исследуемые образцы полупроводниковых лазеров как многомодовые, так и одномодовые показывают существенное обострение выходного оптического импульса, а именно существенное уменьшение полуширины оптического импуль- 
са при неизменной длительности импульса тока накачки. Результаты измерений выходной мощности излучения и полуширины выходного оптического импульса приведены на рис. 1, $a$ (для многомодового лазера) и $b$ (для одномодового лазера).

Из рис. 1, $a$ видно, что многомодовый лазер продемонстрировал максимальную выходную мощность излучения $27 \mathrm{~W}$ и обострение выходного импульса до 120 ps. На вставках приведены осциллограммы импульсов тока накачки и оптических импульсов для значения амплитуды тока накачки 12.1 А. Результаты измерений показали, что генерация оптического импульса начинается на заднем фронте электрического импульса и сдвигается в сторону максимума импульса накачки по мере увеличения его амплитуды. Лазер не проявляет режим срыва генерации во всем диапазоне токов накачки, выходные импульсы излучения при этом однопиковые. При величине тока накачки 12.1 А наблюдается наименьшая полуширина длительности выходного оптического импульса 120 ps. При дальнейшем увеличении тока, прослеживается ее увеличение. Так, при величине тока накачки $35 \mathrm{~A}$ полуширина оптического импульса составляла 280 ps. Из рис. 1 видно, что минимальное значение полуширины оптического импульса соответствует насыщению Ваттамперной характеристики, что может свидетельствовать о влиянии насыщения усиления на работу лазера в данном режиме.

На рис. $1, b$ приведены результаты измерений характеристик излучения одномодового лазера. Видно, что данный образец демонстрирует существенное обострение выходного оптического импульса до 80 ps при токе накачки 2.9 А. При дальнейшем увеличении тока в отличие от многомодового лазера одномодовый лазер проявляет тенденцию к стабилизации величины длительности выходного оптического импульса. Так, величина длительности импульса не превышает 108 ps при токе $7.5 \mathrm{~A}$, а при максимальном значении тока накачки $10 \mathrm{~A}$ длительность выходного оптического импульса составляет $93 \mathrm{ps,} \mathrm{величина} \mathrm{выходной} \mathrm{пиковой} \mathrm{мощности} P$ (она же максимальная) составляет $1.58 \mathrm{~W}$. При токе 2.9 A, соответствующем минимальной длительности импульса, было получено $1.18 \mathrm{~W}$. Минимальное значение полуширины длительности импульса также совпадает с началом загиба ватт-амперной характеристики, т.е., как и в случае многомодового лазера (рис. 1, $a$ ), соответствует насыщению ватт-амперной характеристики. На вставке рис. $1, b$ приведены осциллограммы импульса тока накачки и оптического импульса одномодового лазера для значения амплитуды тока накачки 4.9 А.

Также были исследованы спектральные характеристики одномодовых лазеров. Результаты представлены на рис. 2. Из рисунка видно, что при низких токах накачки лазерная генерация осуществляется только через основное состояние. При величине тока накачки $2.6 \mathrm{~A}$ в спектре излучения появляется еще одна спектральная линия, соответствующая генерации света через вышележащий уровень размерного квантования квантовой ямы,

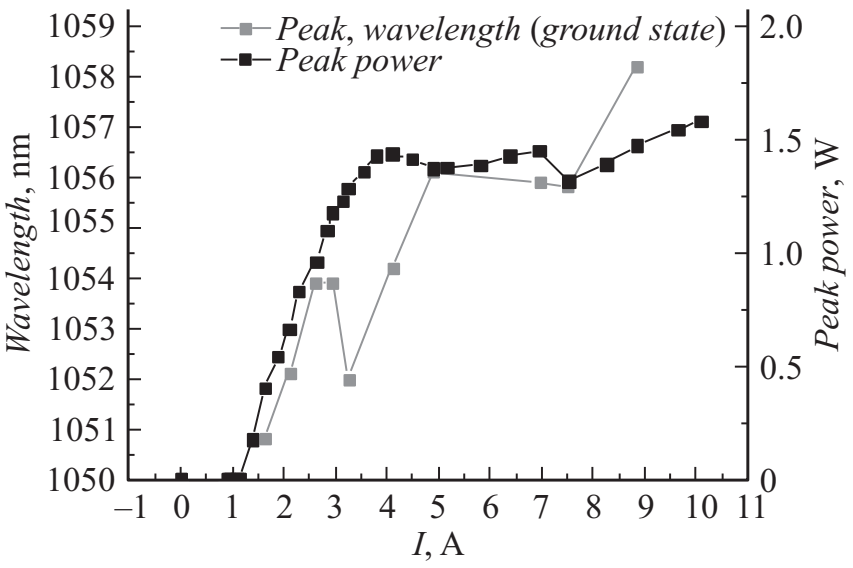

Pис. 3. Зависимости пиковой мощности и длины волны излучения через основное состояние (треугольники) выходного оптического импульса одномодового лазера в зависимости от амплитуды импульса тока накачки длительностью $1 \mathrm{~ns}$.

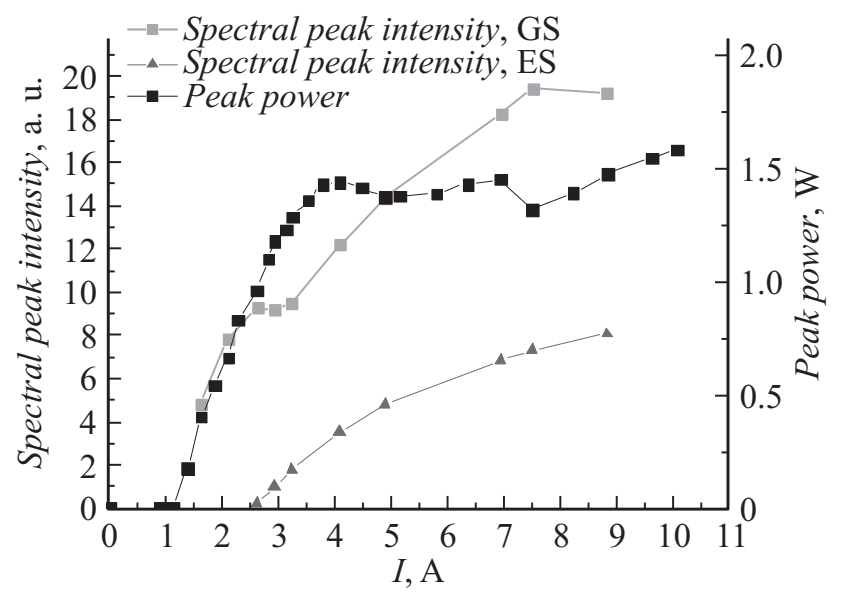

Рис. 4. Зависимости пиковой мощности, спектральной интенсивности излучения через основное состояние, спектральной интенсивности излучения через возбужденное состояние выходного оптического импульса одномодового лазера в зависимости от амплитуды импульса тока накачки длительностью $1 \mathrm{~ns}$.

сдвинутая в коротковолновую область на $86 \mathrm{~nm}$. При токе накачки 9 А интенсивности обеих линий сравниваются. Включение генерации света через вышележащий уровень размерного квантования квантовой ямы $(2.6$, A), последующее начало насыщения Ватт-амперной характеристики (3.6 А), обусловленное, по видимости, насыщением усиления, свидетельствует о связи этих явлений и их влиянии на уменьшение длительности выходного оптического импульса ( 80 ps при токе 2.9 А).

При исследовании выходных спектров одномодового лазера было выявлено, что длина волны, соответствующая генерации с основного состояния, имеет тенденцию к смещению в длинноволновую область (рис. 3), что говорит о том, что несмотря на то, что в эксперимен- 


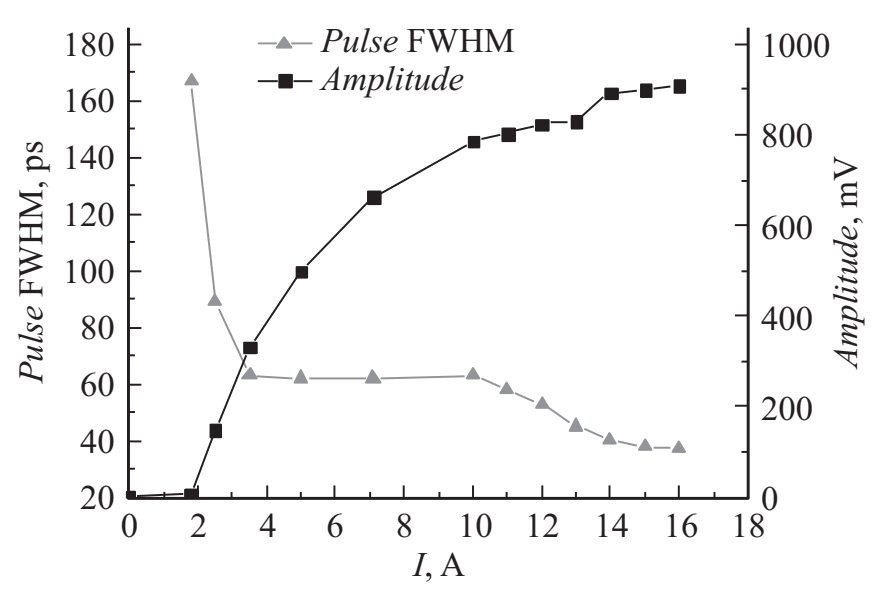

Рис. 5. Зависимости максимума амплитуды и полуширины длительности выходных оптических импульсов одномодового лазера оптического импульса от величины амплитуды импульсов тока накачки длительностью менее $1 \mathrm{~ns}$.

те использовались импульсы тока высокой скважности, происходил нагрев активной области лазера. Величина спектрального сдвига в соответствии с температурной зависимостью ширины запрещенной зоны активной области позволяет оценить величину нагрева активной области на $20-25^{\circ}$.

Если проследить, как изменяется спектральная интенсивность излучения через основное состояние в зависимости от амплитуды тока накачки длительностью $1 \mathrm{~ns}$ (рис. 4), то можно видеть, что наклон этой кривой соответствует наклону кривой выходной пиковой мощности (показана квадратами). При токе накачки, соответствующем появлению в спектре возбужденного состояния (около $2.5 \mathrm{~A}$ ), в зависимости выходной пиковой интенсивности генерации через основное состояние от тока накачки наблюдается излом (англ. „,kink ${ }^{6}$ ), связанный с перераспределением носителей между уровнями и началом генерации с возбужденного состояния. Зависимость максимума интенсивности излучения от величины амплитуды импульса тока накачки через возбужденное состояние показана на этом же рисунке треугольниками.

На рис. 5 представлены данные по измерениям ширины выходных оптических импульсов одномодового лазера от величины тока накачки при уменьшении длительности импульса тока накачки до $0.5 \mathrm{~ns}$. Частота следования импульсов при этом составляла $1 \mathrm{kHz}$. На этом же рисунке приведена зависимость максимума амплитуды оптического импульса от величины тока накачки. Видно, что с увеличением амплитуды импульса тока накачки от 2.0 А длительность выходных импульсов резко уменьшается от $170 \mathrm{ps}$, достигая значения $60 \mathrm{ps}$ при значении амплитуды тока накачки $3.7 \mathrm{~A}$, и в дальнейшем уменьшается до 35 ps при величине амплитуды импульса тока накачки 16 А. С учетом влияния погрешностей измерений, связанных с предельными временами отклика фотоприемника $(\sim 12 \mathrm{ps})$ и осциллографа
( $7 \mathrm{ps})$, оценка минимальной продемонстрированной длительности импульса составляет около $32 \mathrm{ps.}$

Таким образом, в настоящей работе показано, что при короткоимпульсной токовой накачке полупроводниковых лазеров, при определенном подборе параметров накачки, существует режим генерации лазера, при котором возможно получение ультракоротких выходных оптических импульсов с высокой выходной пиковой оптической мощностью. Наблюдаемый эффект обострения лазерных импульсов связан с эффектом насыщения усиления. Было продемонстрировано, что при накачке одномодовых лазеров импульсами тока длительностью менее $1 \mathrm{~ns}$ возможно получить обострение выходного оптического импульса с длительностью до 35 ps.

Работа выполнена при частичной финансовой поддержке РФФИ (грант № 16-32-00332).

\section{Список литературы}

[1] Hou L., Haji M., Marsh J.H. // IEEE J. Select. Topics Quant. Electr. 2013. Vol. 19. N 4. P. 1100808. DOI: $10.1109 /$ JSTQE.2013.2238508.

[2] Huikari J.M.T., Avrutin E.A., Ryvkin B.S., Nissinen J.J., Kostamovaara J.T. // IEEE J. Select. Topics Quant. Electr. 2015. Vol. 21. N 6. P. 1501206. DOI: $10.1109 / \mathrm{JSTQE} .2015 .2416342$.

[3] Ryvkin B.S., Avrutin E.A., Kostamovaara J.T. // J. Lightwave Technol. 2009. Vol. 27. N 12. P. 2125-2131. DOI: $10.1109 /$ JLT.2008.2009075.

[4] Kaltenbach A., Hofmann J., Seidel D., Lauritsen K., Bugge F., Fricke J., Paschke K., Erdmann R., Trankle G. // Proc. SPIE. 2017. Vol. 10088. P. 1008808. DOI: 10.1117/12.2250145.

[5] Fedorova K.A., Sokolovskii G.S., NikitichevD.I., Battle P.R., LivshitsD.A., Rafailov E.U. // Opt. Lett. 2013. Vol. 38. P. 2835-2837. DOI: 10.1364/OL.38.002835.

[6] Sokolovskii G.S., Dudelev V.V., Deryagin A.G., Kuchinskii V.I. // Tech. Phys. Lett. 2012. Vol. 38. N 7. P. 613-615. DOI: 10.1070/QE2013v043n05ABEH015170.

[7] Sokolovskii G.S., Dudelev V.V., Kolykhalova E.D., Deryagin A.G., Maximov M.V., Nadtochiy A.M., Kuchinskii V.I., Mikhrin S.S., Livshits D.A., Viktorov E.A., Erneux T. // Appl. Phys. Lett. 2012. Vol. 100. P. 081109. DOI: $10.1063 / 1.3688604$.

[8] Viktorov E.A., Erneux T., Kolykhalova E.D., Dudelev V.V., Danckaert J., Soboleva K.K., Deryagin A.G., Novikov I.I., Maximov M.V., Zhukov A.E., Ustinov V.M., Kuchinskii V.I., Sibbett W., Rafailov E.U., Sokolovskii G.S. // Phys. Rev. E. 2016. Vol. 94. P. 052208. DOI: 10.1103/PhysRevE.94.052208.

[9] Sokolovskii G.S., Cataluna M.A., Deryagin A.G., Kuchinskii V.I., Novikov I.I., Maximov M.V., Zhukov A.E., Ustinov V.M., Sibbett W., Rafailov E.U. // Tech. Phys. Lett. 2007. Vol. 33. P. 4-7. DOI: 10.1134/S1063785007010026.

[10] Sokolovskii G.S., Viktorov E.A., Abusaa M., Danckaert J., Dudelev V.V., Kolykhalova E.D., Soboleva K.K., Deryagin A.G., Novikov I.I., Maximov M.V., Zhukov A.E., Ustinov V.M., Kuchinskii V.I., Sibbett W., Rafailov E.U., Erneux T. // Appl. Phys. Lett. 2015. Vol. 106. P. 261103. DOI: $10.1063 / 1.4923244$. 
[11] Sokolovskii G.S., Vinokurov D.A., Deryagin A.G., Dudelev V.V., Kuchinskii V.I., Losev S.N., Lyutetskiy A.V., Pikhtin N.A., Slipchenko S.O., Sokolova Z.N., Tarasov I.S. // Tech. Phys. Lett. 2008. Vol. 34. N 8. P. 708-710. DOI: $10.1134 / \mathrm{S} 1063785008080257$.

[12] Efanov V.M., Kriklenko A.V., Yarine P.M. // IEEE Conf. on Pulsed Power Plasma Sci. 17-22 June 2001. DOI: 10.1109/PPPS.2001.961232.

[13] Dudelev V.V., Zazulin S.V., Kolykhalova E.D., Losev S.N., Deryagin A.G., Kuchinskii V.I., Efanov M.V., Sokolovskii G.S. // Tech. Phys. Lett. 2016. Vol. 42. N 12. P. 1159-1162. DOI: 10.1134/S1063785016120191.

[14] Portnoi E.L., Venus G.B., Khazan A.A., Gadjiev I.M., Shmarcev A.Yu., Frahm J., Kuhl D. // IEEE J. Select. Topics Quant. Electr. 1997. Vol. 3. P. 256-260. DOI: $10.1109 / 2944.605666$.

[15] Lanz B.1., Ryvkin B.S., Avrutin E.A., Kostamovaara J.T. // Opt. Exp. 2013. Vol. 21. N 24. P. 29780-29791.

DOI: 10.1364/OE.21.029780. 